\title{
LEUCEMIA MIELOIDE CRÓNICA(PH+): DIAGNÓSTICO Y TRATAMIENTO
}

\author{
García Fallas, Miriam
}

Médico General, Servicio de Urgencias de la Clínica Marcial Fallas Díaz, San José, Costa Rica

Resumen:La Leucemia Mielode Crónica es considerada como un modelo para el desarrollo de terapias de blanco molecular debido al conocimiento de su patogénesis. Esta enfermedad se caracteriza por la presencia del cromosoma Philadelfia $(\mathrm{Ph}+$ ) producto de la traslocación recíproca entre los cromosomas 922 originando el gen Bcr-Abl. La presencia de la oncoproteía producto de la expresión de este gen en las células permite la transformación leucémica con una marcada sensibilidad disminuida a la regulación permitiendo la expansión clonal, interviniendo en los mecanismos de diferenciación y activando vías de supervivencia. El desarrollo del Imatinib para su tratamiento bloqueando la acción de la oncoproteína, ha permitido la aplicación de diversas técnicas de biología molecular para definir parámetros de respuesta y progresión de dicha enfermedad lo que ha influido en las tomas de decisión oportunas en cuanto a cambios terapéuticos en pacientes con riesgo de progresión que se asocia a resistencia a Imatinib, así como en su seguimiento en pacientes con respuesta molecular completa y mayor.

Palabras clave: Leucemia Mieloide Crónica, Imatinib, monitoreo, transcriptos Abl-Bcr 


\section{LEUKEMIA (PH +): DIAGNOSIS AND TREATMENT}

Abstract: Chronic myelogenous leukemia is considered as an exemplary for the development of molecular targeted therapies due to its pathogenesis. This disease is characterized by the presence of Philadelphia chromosome (PH+), a product of the reciprocal translocation between chromosomes 9 and 22, which causes the BCR-Abl gene. The appearance of this gene in the cells gives rise to an oncoprotein that allows a leukemic transformation with a marked decreased sensitivity to regulation letting clonal expansion manifest. In addition, this oncoprotein intervenes in myriad mechanism of differentiation and activates survival pathways. The development of Imatinib treating this disease blocks the action of the oncoprotein and puts into practice different techniques of molecular biology in order to define parameters of response and progression of such disease; this allows decision making regarding therapeutic changes in patients who are at risk of progression associated to Imatinib resistance, and it monitors patients with a complete and higher molecular.

Key words: Chronic Myeloid Leukemia, Imatinib, tracking, Alb-Bcr transcriptions

\section{INTRODUCCIÓN}

La Leucemia Mieloide Crónica (LMC) puede considerarse como un paradigma dentro de las enfermedades neoplásicas, debido al conocimiento de su patogénesis se considera como una de las enfermedades modelo para el desarrollo de las terapias dirigidas a blancos moleculares para su tratamiento [1].

Es una enfermedad maligna de origen hematológico que se caracteriza por un cuadro de origen insidioso como se resume en la tabla 1 [2,16]: Estas células presentes en médula ósea y en sangre periférica contienen una traslocación recíproca entre los cromosomas 9 y 22 en más del $90 \%$ de los pacientes, conocida como cromosoma Philadelfia (Ph) [2].

Este fenómeno de traslocación recíproca implica el intercambio de material entre estos dos cromosomas originando el gen Bcr-Abl [2, 17].

El gen Bcr-Abl se origina de la traslocación que yuxtapone el extremo $3^{\prime}$ de la secuencia del protooncogén Abl del cromosoma 9 con la secuencia 5' del gen Bcr del cromosoma 22 [3].

La historia natural de la enfermedad se caracteriza por 3 fases como se describe en la tabla 2 $[1,2,3]$.
Tabla 1. Hallazgos clínicos y manifestaciones hematológicas de la Leucemia Mieloide Crónica

\begin{tabular}{|c|c|}
\hline Hallazgos clínicos & $\begin{array}{l}\text { Manifestaciones } \\
\text { hematológicas }\end{array}$ \\
\hline $\begin{array}{l}\text { Fatiga, pérdida de } \\
\text { peso }\end{array}$ & $\begin{array}{l}\text { Leucocitosis }\left(150 \times 10^{9} / \mu \mathrm{l}\right) \\
\text { con formas maduras e } \\
\text { inmaduras de granulocitos. } \\
\text { Basofilia, eosinofilia y } \\
\text { monocitosis }\end{array}$ \\
\hline Esplenomegalia & $\begin{array}{l}\text { Menos de un } 5 \% \text { son formas } \\
\text { blásticas }\end{array}$ \\
\hline Infecciones & Trombocitosis \\
\hline $\begin{array}{lr}\text { Trombosis } & \text { como } \\
\text { enfermedad } & \\
\text { vasooclusiva } & \text { o } \\
\text { hemorragias } & \end{array}$ & $\begin{array}{ll}\text { Anemia } & \text { normocrómica } \\
\text { normocítica }\end{array}$ \\
\hline $\begin{array}{lr}\text { Accidentes } & \text { cerebro } \\
\text { vasculares, } & \text { infarto } \\
\text { miocárdico, } & \\
\text { priapismo, dolor óseo }\end{array}$ & $\begin{array}{l}\text { Médula ósea: celularidad } \\
\text { aumentada, con porcentaje } \\
\text { de blastos normal o } \\
\text { levemente aumentados. } \\
\text { Al inicio es poco frecuente la } \\
\text { fibrosis colágena. }\end{array}$ \\
\hline
\end{tabular}




\section{Tabla 2. Fases de la Leucemia Mieloide Crónica}

\begin{tabular}{|ll|}
\hline Fase crónica & Estado inicial de la Leucemia. \\
& $\begin{array}{l}\text { Insidiosa. } \\
\text { Es cuando en la mayor parte se } \\
\text { realiza el diagnóstico }\end{array}$ \\
\hline $\begin{array}{l}\text { Fase de } \\
\text { aceleración }\end{array}$ & Etapa refractaria a la terapia. \\
& $\begin{array}{l}\text { Aumento de la esplenomegalia y del } \\
\text { número de blastos. }\end{array}$ \\
& Trombocitopenia. \\
\hline $\begin{array}{l}\text { Crisis } \\
\text { Blástica }\end{array}$ & $\begin{array}{l}\text { Empeoramiento del estado clínico. } \\
\text { Presencia de infiltración blástica en } \\
\text { médula ósea y } \\
\text { extramedular }\end{array}$ \\
\end{tabular}

En su epidemiología podemos anotar que en Estados Unidos la Leucemia Mieloide crónica representa aproximadamente un $15 \%$ de todos los casos de Leucemia, con unos 4600 nuevos casos por [2].

Es más frecuente en hombres $(1,5: 1,0)$ pero las manifestaciones clínicas y la evolución de la enfermedad no muestra diferencias significativas. La incidencia es de 2 por 100000 habitantes, aumentando esta exponencialmente con la edad. Y aproximadamente 1600 personas mueren anualmente [2].

En Costa Rica las leucemias se encuentran dentro de las neoplasias más frecuentes con una tasa de mortalidad de 23,8 por 100000 en hombres y de 19,5 por 100000 en mujeres. Se observa a demás un ligero predominio del sexo masculino en la tasa de mortalidad y en los egresos hospitalarios [4].

\section{ETIOLOGÍA Y PATOGÉNESIS}

La LMC es la enfermedad maligna más estudiada, y es la primera en ser relacionada con una alteración cromosómica, el cromosoma Philadelfia $(\mathrm{Ph})$.

El cromosoma Ph puede aparecer después del evento leucémico inicial, incluso algunos pacientes han experimentado periodos de enfermedad cuando el cromosoma $\mathrm{Ph}$ ha desaparecido [2].

\section{FACTORES DE RIESGO}

El factor más importante es la exposición a radiación ionizante, donde la incidencia de LMC se ha demostrado aumenta en las siguientes poblaciones: exposición a radiación de los sobrevivientes de la bomba atómica, pacientes con espodilitis anquilosante que recibieron radioterapia y pacientes con cáncer de cuello uterino [5].

\section{PATOGÉNESIS DE LA LMC}

La transformación leucémica de la fusión de los genes Abl-Bcr, producto de la traslocación ya descrita, es mantenida por un pequeño número de células madre que favorecen este linaje.

Esta predisposición hacia la diferenciación y a la expansión de la célula progenitora está mediada por un mecanismo de control autocrino a través de la Interleucina 3 (IL-3) y Factor Estimulante de los Granulocitos (G-CSF) [5].

Las células progenitoras tienen la capacidad marcada de expansión clonal según su linaje: eritroide, granulocítico, megacariocítico, estas poblaciones celulares tienen una sensibilidad disminuida a la regulación. La marcada expansión de los granulocitos en sangre resulta del aumento del proceso granulopoyético y en menor contribución de un tiempo mayor de circulación intravascular [5].

La localización citoplasmática de la oncoproteína Bcr-Abl permite el acceso a substratos celulares que no estaban antes disponibles por la localización nuclear de la proteína Abl, determinando la activación de las vías de supervivencia, la detención del proceso de diferenciación, de proliferación clonal y la incapacidad para detectar daños en el genoma y su reparación [3,5,6,18]. 


\section{Tabla 3. Alteraciones genéticas más importantes}

\begin{tabular}{|c|c|}
\hline & Mecanismo Afectado \\
\hline \multirow[t]{2}{*}{$\begin{array}{l}\text { Detención } \\
\text { proceso } \\
\text { diferenciación }\end{array}$} & $\begin{array}{l}\text { Supresión del factor de } \\
\text { transcripción CEBP } \\
\text { enhancer-binding } \\
\text { alpha) }\end{array}$ \\
\hline & $\begin{array}{l}\text { Activa la transcripción del } \\
\text { receptor del factor estimulante } \\
\text { de los granulocitos (GCSFR) y } \\
\text { del gen ID1. }\end{array}$ \\
\hline $\begin{array}{llr}\text { Pérdida } & & \text { de } \\
\text { vigilancia } & & \text { de } \\
\text { errores } & \text { en } & \text { el } \\
\text { genoma } & & \end{array}$ & $\begin{array}{l}\text { ATM/ATR } \\
\text { BCRA } 1\end{array}$ \\
\hline \multirow[t]{3}{*}{$\begin{array}{l}\text { Deficiencia en la } \\
\text { reparación. }\end{array}$} & $\begin{array}{l}\text { NHEJ (non homologous end- } \\
\text { joing) }\end{array}$ \\
\hline & $\begin{array}{l}\mathrm{HR} \\
\text { recombination) (homologous }\end{array}$ \\
\hline & $\begin{array}{l}\text { NER (Nucleótido excision } \\
\text { repair) }\end{array}$ \\
\hline $\begin{array}{l}\text { No activación de la } \\
\text { Apoptosis. }\end{array}$ & $\begin{array}{l}\text { Bloqueo de la Activación de la } \\
\text { Caspasa } 3\end{array}$ \\
\hline
\end{tabular}

La inestabilidad genómica también es una característica de las vías afectadas por la oncoproteína. A través de inducir mutaciones y fallos en el mecanismo de detección de daños y reparación de $\operatorname{ADN}[3,5,6,18]$.

Esto podría explicar la aparición de anormalidades cromosómicas como trisomía 8, un cromosoma $\mathrm{Ph}$ adicional, isocromosoma 17, trisomía 19, pérdida del cromosoma Y en hombres, trisomía 21, y monosomía 7, estos cambios han sido utilizados como marcadores de progresión de enfermedad. [3].

Otros de los aspectos importantes es la pérdida de la vigilancia de los errores en el genoma. En este sentido se hace énfasis en las proteínas ATR (Ataxia telangectasia and Rad 3 protein)y ATM (Ataxia telangiectasia mutated protein). Las cuáles son proteínas cinasas que se considera poseen la función de sensores de daños en el ADN lo que permite a partir de una cadena de fosforilaciones regular el paso de la fase S a G1 del ciclo celular, al no estar reguladas adecuadamente hay una replicación inadecuada del ADN a pesar del daño $[3,5,6,18]$.

Otros de los genes involucrados por BRCA 1 (BRCA 1 associated genome surveillance complex). Luego del daño al ADN, BRCA 1 organiza diferentes sensores y coordina la actividad de reparación. BRCA 1 en LMC se encuentra en concentraciones bajas y está en regulación disminuida (down regulation) [3,5,6,18].

Otro mecanismo que se encuentra alterado es el NER (Nucleotido excision repair); éste involucra la reparación del ADN cuando sufre lesiones que se describen como "bulky" o alteraciones en la hélice. NER reconoce la lesión y corta dentro de un rango de 25-30 nucleótidos, reparando luego con la síntesis de ADN. En la LMC las deficiencias en NER podrían aumentar la inestabilidad genómica que caracteriza la fase avanzada de la enfermedad. $[3,5,6,18]$.

Otro aspecto importante es el defecto en el acortamiento de telómeros que ocurre en las leucemias. Existe una asociación entre el acortamiento de los telómeros en las enfermedades neoplásicas y un aumento en la inestabilidad genómica y de una mayor predisposición a la progresión de la enfermedad, por efecto en la supervivencia de la célula [7].

\section{RESISTENCIA A IMATINIB}

\section{IMATINIB: CARACTERÍSTICAS GENERALES $Y$ MECANISMO DE ACCIÓN}

El desarrollo del Imatinibmesilato se estimuló gracias al conocimiento de las vías y las funciones de la oncoproteínaBcr-Abl [1].

En la década de los 90, se inicia el proyecto de sintetizar una molécula que sea capaz de inhibir la actividad tirosíncinasa de la oncoproteína a través de la competencia por el ocupar el sitio de unión al ATP [1].

El mecanismo de acción del Imatinib es competir por este sitio de unión. La oncoproteína puede catalizar la fosforilación de los residuos de tirosina en las proteínas substratos. El imatinib es estructuralmente similar al ATP y puede unirse al sitio de unión de éste dentro del dominio de Bcr-Abl [1].

El imatinib no tiene grupos fosfatos disponibles para ser transferidos al sustrato por lo que la

Revista electrónica publicada por el Departamento de Farmacología de la Escuela de Medicina de la Universidad de Costa Rica, 2060 San José, Costa Rica. ${ }^{\circledR}$ All rights reserved. Licensed under a Creative Commons Unported License. 
fosforilación no se lleva a cabo. Las proteínas sustratos de la oncoproteína al no estar fosforiladas no pueden adoptar la conformación necesaria para unirse a las moléculas efectoras y entonces se da una señalización corriente abajo, la señalización es inhibida como consecuencia $[1,19$,$] .$

El uso del imatinib desplazó al transplante de médula ósea como tratamiento primario de elección en estos pacientes, además de brindar una opción terapéutica a los pacientes que no respondían a citarabina y al interferón alfa $[8,19,23]$.

\section{DEFINICIÓN DE RESISTENCIA}

Se define la resistencia primaria como un fallo para obtener una respuesta hematológica completa (cuantificación de Bcr-abl por PCR) a pesar de un aumento en la dosis de Imatinib, esto ocurre aproximadamente en $5 \%$ de los casos $[9,18$,$] .$

En el 15\% de los casos en fase crónica ocurre la resistencia citogénetica primaria definida como el fallo en obtener una respuesta citogenética mayor (menos de $35 \%$ de metafases $\mathrm{Ph}$ positivas en biopsia de médula ósea) 6 meses después de la terapia o 12 meses después de esta [9].

El otro tipo es la secundaria o la resistencia hematológica o citogenética adquirida, refiriéndose a la pérdida de una respuesta establecida previamente [9].

Luego de 42 meses de seguimiento un $16 \%$ de los pacientes en fase crónica desarrolla una resistencia secundaria o progresión de enfermedad [9].

\section{MECANISMO DE RESISTENCIA DEPENDIENTE DE BCR-ABL}

El mayor mecanismo de resistencia a imatinib es la expansión clonal de células leucémicas con mutaciones en la tirosín cinasa que reduce la capacidad del imatinib de inhibir la actividad cinasa [10].

Este mecanismo de resistencia se presenta entre un 50 a un $90 \%$ de los casos. Se han encontrado más de 40 diferentes mutaciones que han sido asociadas a resistencia clínica a imatinib $[10,19]$.
La resistencia se asocia a mutaciones que ocurren en los aminoácidos implicados directamente a la unión con el imatinib, la mayoría previene que el dominio de la cinasa adopte la conformación específica para que éste logre entrar al sitio de unión ATP $[9,19,20,21,22]$.

Aproximadamente el $10 \%$ de la resistencia se asocia a una sobreproducción de Bcr-Abl o a la adquisición de un cromosoma $\mathrm{Ph}$ adicional. Se presume entonces que la concentración intracelular de Imatinib es insuficiente para inhibir la oncoproteína. [9].

\section{MECANISMO DE RESISTENCIA INDEPENDIENTE DE BCR-ABL}

Se ha reportado una sobreexpresión de señales relacionadas con los mecanismos de regulación de la transcripción y traducción, sugiriendo vías independientes a la actividad cinasa de la oncoproteína Bcr-Abl, que también confiere resistencia a imatinib $[11,21,22]$.

\section{MONITOREO DE LOS PACIENTES $Y$ LA IMPORTANCIA DE LA CUANTIFICACIÓN DE LOS TRANSCRIPTOS ABL-BCR}

El primer estudio que demostró la eficacia del tratamiento con Imatinib y la importancia de la cuantificación de los transcriptos Bcr-Abl, fue: The International Randomised Study of Interferon and ST1571 (IRIS) [13, 14].

El objetivo del estudio era reducir la carga de células leucémicas y asociar este valor con periodo libre de enfermedad y progresión de la enfermedad. En los primeros 12 meses más del $95 \%$ de los pacientes tratados con Imatinib tuvieron una respuesta hematológica completa y el $85 \%$ de estos tuvieron una respuesta molecular completa $[13,14]$.

Este estudio identificó que una reducción estricta de la carga leucémica de un orden de magnitud por debajo de la respuesta citogenética completa, confería un pronóstico superior de casi un $100 \%$ de disminución del riesgo de progresión de la enfermedad. $Y$ esto fue definido como respuesta molecular mayor (MMR) y representa 3 log de reducción de los niveles de Bcr-Abl medidos pretratamiento $[13,14]$. 
La técnica de PCR puede utilizarse para el diagnóstico al detectar los transcriptos de Bcr-Abl, además puede ser usada para confirmar los hallazgos citogenéticos en casos con variantes de rearreglos en el cromosoma $\mathrm{Ph}$ cuya interpretación en el cariotipo es difícil de lograr [13, 14,19,20,21].

El monitoreo de RQ-PCR es considerado una parte integral del manejo de pacientes tratados con Imatinib y el fallo de alcanzar una respuesta molecular mayor en un periodo de 18 meses después de iniciado el tratamiento, es considerada como una respuesta subóptima que requiere una cuidadosa reevaluación y una readecuación terapéutica $[15,19,20,21]$.

El criterio de respuesta molecular completa se define usando de forma estricta el criterio de sensibilidad probablemente ayuda a identificar pacientes con respuestas durables incluso luego de la descontinuar el uso de imatinib [15,20,21,22].

Una carga indetectable de Bcr-Abl no necesariamente significa la erradicación de la enfermedad. Sin embargo, se ha sugerido que el monitoreo se debe llevar a cabo cada 3 meses en los pacientes que han alcanzado una respuesta molecular mayor $[15,20,21$,

Si se confirman aumentos secuenciales en los niveles de Bcr-Abl, se podría ayudar a identificar de forma temprana un riesgo de recaída, progresión o indicativo de posibles mutaciones en la oncoproteína. [15].

Finalmente la determinación de los transcriptos es fundamental para monitorear pacientes con respuesta citogenética completa de forma complementaria $[15,19,20,21,22]$.

\section{CONCLUSIONES}

La Leucemia Mieloide Crónica (LMC) puede considerarse como un paradigma dentro de las enfermedades neoplásicas, debido al conocimiento de su patogénesis es una de las enfermedades modelo para el desarrollo de las terapias dirigidas a blancos moleculares para su tratamiento.

Se caracteriza por la presencia del cromosoma Filadelfia, el cuál es producto de la traslocación del cromosoma 9 y 22 dando origen a una oncoproteína capaz de interferir en los procesos de diferenciación, aumentar los mecanismos de sobrevivencia y expansión clonal lo que resulta en las manifestaciones clínicas que podemos observar en los pacientes con Leucemia Mieloide Crónica.

El imatinib es el fármaco que nace a partir del conocimiento de las funciones de la oncoproteína, y es capaz de inhibir actividad tirosín cinasa que le permite intervenir en los procesos que explican la patogénesis de la enfermedad.

El mayor mecanismo de resistencia a imatinib es la expresión clonal de células con trasformación leucémica que presenten mutaciones en la tirosín cinasa la cual previene que el dominio cinasa adopte la conformación para que éste logre entrar al sitio de unión del ATP.

A partir del estudio IRIS (The International Randomised Study of Interferon and ST1571) se demuestra la importancia de cuantificar los transcriptos del oncogen Bcr-Abl para poder establecer una relación entre el periodo libre de enfermedad y progresión de la enfermedad.

Pero además esta cuantificación es considerada una parte integral en el manejo de los pacientes tratados con imatinib y el fallo de alcanzar una respuesta molecular mayor en un periodo de 18 meses después de iniciado el tratamiento lo que permite considerarlo como una respuesta subóptima que requiere una cuidadosa reevaluación y una readecuación del tratamiento médico.

\section{REFERENCIAS}

1. Barnes D,Melo, J. Cytogenetic and molecular genetic aspects of chronic myeloid leukaemia. Ac. Haemat. 2010; 8(4): 180-202.

2. Lichtman M. Acute myelogenous leukemia. En. Williams Hematology. McGraw-Hill, New York , United States, 7 Th Edition, 2007, pp. 1183-1236.

3. Melo J,Barnes D. Chronic myeloid leukaemia as a model of disease evolution in human cancer. Nat. Rev. Can. 2007 June 7(6): 441-453.

4. Madrigal, L. 2005. Variaciones cronológicas y geográficas del cáncer de pulmón, piel y otros de menor frecuencia 1956-57 a 2002-2003. CCSS, http://www.ccss.sa.cr/html/organizacion/gestión/g 
erencias/medica/germed/dis/diess/ca piel.htmAcce sada el 28 de mayo 2012.

5. Marshall A, Beutler E, Kipps T, Seligsohn U, Kaushansk, K,Prchal T. Hematology. McGrawHill . New York, United States, 2006, pp. 1237-1294.

6. Jiménez G, J. Carrillo, M, Chaves R, Jiménez M, Vargas L, Campos A, et al. Detección molecular del gen BcrAbl por RT- PCR en niños costarricenses con leucemia. Rev. Biol. Trp. 2006; 56(4): 1613-1618.

7. Gordon M, Dazzi F, Marley S, Lewis J, Nguyen D, Grand F, et al. Cell biology of CML cells. Leukemia: Off. J. of Leuk. Soc. Of Am. 1999;13 (suppl 1): s65s71.

8. Giralt S,Arora M, Goldman J, Lee S,Maziarz R, McCarthy $\mathrm{P}$, et al. Impact of imatinib therapy on the use of allogeneic haematopoietic progenitor cell transplantation for the treatment of chronic myeloid leukaemia. Brit. J. of Haemat. 2007;137(5): 461-467.

9. Shah N. Loss of response to Imatinib: Mechanisms and management. Hematology. 2005;33 (9): 183187.

10. Brandfor S, Hughes T. Myeloid Leukemia: methods and Protocols. Humana press. New Jersey, USA. 2006, pp. 93-105.

11. Breccia M. Alimena G. Resistance to Imatinib in chronic myeloid leukemia and therapeutic. Approaches to circumvent the problem. Cardio. Haemat. Dis. Drug. Targ. 2009;9(1): 21-28.

12. Ross D, Branford S, Moore S, Hughes T. Limited clinical value of regular bone marrow cytogenetic analysis in imatinib-treated chronic phase CML patients monitored by RQ-PCR for BCR-ABL. Leuk.: Off. J. Of Leuk. Soc. of Am. 2006;20(4): 664-670.

13. Ross D, Hughes T. Current and emerging tests for the laboratory monitoring of chronic myeloid leukaemia and related disorders. 2008;40(3): 231246.

14. Mauro, M. Defining and Managing Imatinib Resistance. Haematology. 2006 (1)219-225.

15. Baccarani F, Saglio P. Monitoring treatment of chronic myeloid leukemia. Haematologica. 2008; 93(2): $161-166$.

16. Wetzel, M, Byrd, J, Bloomfield, C. Leucemia mieloide aguda y crónica. En. Harrison Principios de Medicina Interna. McGraw-Hill, México, D.F, México, 17 Th Edition, 2008, pp. 677-686.
17. Hassold, T, Schwarzt, S. Trastornos cromosómicos. En. Harrison Principios de Medicina Interna. McGraw-Hill, México, D.F, México, 17 Edición, 2008, pp. 412.

18. Rumpold, H, Webersinke,G. Molecular pathogenesis of Phidelphia positive chronic myeloid leukemia-is it all BCR-ABL? Curr Cancer Drug Tar. 2011;11(1):319.

19. Tefferi A, Dewald G, Litzow M, Cortes J, Mauro M, Kantarjian $\mathrm{H}$, et al. Chronic myeloid leukemia: current application of cytogenetics and molecular testing for diagnosis and treatment. Mayo Clin. Proc. 2005;80(3):390-402.

20. Sessions J. Monitoring your patients with chronic myeloid leukemia. Am. J. Health-Syst, 2006;63 (23 suppl 8): S5.

21. Thijsen S, Schuurhuis G, van Oostveen J, Ossenkoppele G. Chronic myeloid leukemia from basics to bedside. Off. J. Leu. Soc. Of Am. 1999; 13(11): 1646-1674.

22. Takahashi N, Miura M. Therapeutic drug monitoring of imatinib for chronic myeloid leukemia patients in the chronic phase. Pharmacology. 2011;87(5-6): 241-248.

23. Simonsson B, Hjorth-Hansen H, Bjerrum O, Porkka K. Interferon alpha for treatment of chronic myeloid leukemia. Curr. Drug, Tar. 2011;12(3): 420-428.

\section{INFORMACION DE AUTOR:}

\author{
García Fallas, Miriam \\ E-mail: garciafallas@yahoo.com \\ Clínica Marcial Fallas, San José, Costa Rica.
}

